\title{
Impactos normativos y mediáticos de los casos de agresiones con ácido a mujeres en España y Colombia 2010-2017
}

\section{Regulatory and media impacts of cases of attacks with acid on women in Spain and Colombia 2010-2017}

https://doi.org/10.15332/iust.v0i16.2399

\author{
Sheila Giraldo Duque \\ Magister en Derecho con énfasis en Derechos Humanos y Justicia Transicional, Universidad del Rosario, Bogotá; \\ Comunicadora Social, Fundación Universitaria UNINPAHU, Bogotá. Asistente de Investigación Grupo de \\ Investigación en Derechos Humanos, Facultad de Jurisprudencia, Universidad del Rosario. \\ Correo electrónico: sheila.giraldo@urosario.edu.co
}

\begin{abstract}
Resumen
El presente documento expone los resultados de la investigación: "Estudio comparado de los impactos normativos y mediáticos de las agresiones con ácidos a mujeres sobrevivientes en España y Colombia", la cual tuvo por objeto abordar la situación de estas agresiones con el propósito de responder a la pregunta sobre cómo ha sido el tratamiento noticioso de la temática en los dos países y su influencia en la estructuración y fortalecimiento de sistemas jurídicos. De esta manera, a partir de la implementación de una metodología de investigación cualitativa, se adelantó una revisión normativa y documental que permitió la selección de dos casos paradigmáticos, en los dos países, a partir de los cuales fue posible identificar impactos de la esfera mediática en escenarios de la vida y agenda pública, como: la influencia positiva de las prácticas comunicativas en el desarrollo normativo y jurisprudencial de los Estados, su incidencia en las decisiones judiciales, la regulación de la comercialización de sustancias químicas y la visibilidad de las organizaciones civiles que, lideradas en su mayoría por sobrevivientes, buscan la garantía de sus derechos. Finalmente, este trabajo presenta unas conclusiones en las que resalta la importancia del reconocimiento de la influencia de la esfera mediática en el tratamiento de las agresiones con ácidos en escenarios que resultan en la revictimización de esta población, víctima de una problemática que actualmente trasciende fronteras y al género femenino.
\end{abstract}

Palabras clave: Esfera mediática, agresiones con ácido, mujeres, víctimas, sobrevivientes, opinión pública, legislación, derechos humanos.

\begin{abstract}
Summary
This document presents the results of the research: Comparative study of the normative and media impacts of acid aggressions on surviving women in Spain and Colombia, which aimed to address the situation of these aggressions in order to answer the question on how the news treatment of the subject has been in the two countries and its influence on the structuring and strengthening of legal systems. In this way, from the implementation of a qualitative research methodology, a normative and documentary review was carried out that allowed the selection of two paradigmatic cases, in the two countries, from which it was possible to identify impacts of the media sphere in life scenarios and public agenda, such as: the positive influence
\end{abstract}

Recibido: 20 de marzo de 2018, Aprobado: 15 de septiembre de 2018 . 
of communicative practices in the normative and jurisprudential development of States, their impact on judicial decisions, the regulation of the commercialization of chemical substances and the visibility of civil organizations which, led mostly by survivors, seek the guarantee of their rights. Finally, this paper presents some conclusions that highlight the importance of the recognition of the influence of the media sphere in the treatment of aggressions with acids in scenarios that result in the re-victimization of this population, victim of a problem that currently transcends Borders and the female gender.

Keywords: Media sphere, acid attacks, women, victims, survivors, public opinion, legislation, human rights.

\section{Résumé}

Ce document présente les résultats de la recherche: Étude comparative des impacts normatifs et médiatiques des agressions à l'acide sur les femmes survivantes en Espagne et en Colombie, qui visait à remédier à la situation de ces agressions afin de répondre à la question sur la manière dont le sujet a été traité dans les deux pays et son influence sur la structuration et le renforcement des systèmes juridiques. Ainsi, à partir de la mise en œuvre d'une méthodologie de recherche qualitative, une revue normative et documentaire a été réalisée qui a permis de sélectionner deux cas paradigmatiques, dans les deux pays, parmi lesquels il était possible d'identifier les impacts de la sphère des médias. dans les scénarios de vie et les priorités publiques, tels que: l'influence positive des pratiques de communication sur le développement normatif et jurisprudentiel des États, leur impact sur les décisions de justice, la réglementation de la commercialisation des substances chimiques et la visibilité des organisations de la société civile qui, dirigés principalement par les survivants, cherchent la garantie de leurs droits. Enfin, cet article présente des conclusions qui soulignent l'importance de la reconnaissance de l'influence de la sphère médiatique dans le traitement des agressions à l'acide dans des scénarios qui aboutissent à la revictimisation de cette population, victime d'un problème qui transcende actuellement Les frontières et le genre féminin.

Mots-clés: Sphère médiatique, attaques à l'acide, femmes, victimes, survivants, opinion publique, législation, droits de l'homme. 


\section{Impactos normativos y mediáticos de los casos de agresiones con ácido a mujeres en España y Colombia 2010-2017 ${ }^{1}$}

Sheila Giraldo Duque

\section{INTRODUCCIÓN}

En la actualidad, las agresiones con ácido se manifiestan como una de las principales formas de violencia extrema, que ubican a Bangladesh, Camboya, India, Pakistán y Colombia como aquellos países que más casos registran. De forma complementaria, actualmente se cuenta con hallazgos que sitúan a Inglaterra como el foco de estas agresiones en el continente europeo.

Es por lo que, en el contexto de estas agresiones, el presente trabajo pretende responder a la pregunta de cómo ha sido el tratamiento noticioso del tema de estudio en dos países específicamente: España y Colombia, para finalmente identificar los impactos de la esfera mediática en escenarios de la agenda pública en los que se desarrolla este asunto.

A pesar de que los hallazgos evidencian que a nivel mundial las agresiones con ácido han dejado de ser un tema característico de la violencia de género, este trabajo aborda esta problemática a partir de la elección de dos casos en los que las sobrevivientes son mujeres. Esta razón obedece, principalmente, a que los hallazgos demuestran que ha sido precisamente la existencia y difusión mediática de casos particulares en los que la población agredida corresponde al género femenino, lo que ha promovido el desarrollo normativo y jurisprudencial de los Estados.

Es así como este trabajo abordará las agresiones con ácido como una forma de violencia extrema contra las mujeres, entendida, desde la definición de Osorio (2015) citado en Villalobos (2017), como una "forma de acción específica, un fenómeno social particular que parece situarse en un más allá de lo concebible y lo aceptable de la violencia". Esto es, "una categoría de crímenes no solo particularmente graves, sino también diferentes en cuanto a su significado en el campo de las otras prácticas de la violencia". De esta manera, las agresiones con ácido o agentes químicos constituyen todo acto de violencia basado en la pertenencia al sexo femenino que tenga o pueda tener como resultado un daño o sufrimiento físico, sexual o sicológico para la

1 El presente texto es producto del trabajo investigativo en la Maestría en Derecho con énfasis en Derechos Humanos. Estudio comparado de los impactos normativos y mediáticos de las agresiones con ácidos a mujeres sobrevivientes en España y Colombia. 
mujer, así como las amenazas de tales actos (...), tanto si se producen en la vida pública como en la vida privada (Declaración sobre la eliminación de la violencia contra la mujer, 1993).

Finalmente, una de las motivaciones principales que promueven el desarrollo de este trabajo académico, obedece a la importancia de reconocer que, además de los Estados, los medios de comunicación son un actor determinante en la promoción de los derechos humanos y en el restablecimiento de los derechos de las víctimas o sobrevivientes de cualquier tipo de violencia.

Es a través de prácticas comunicativas particulares que en países como los que son objeto de estudio, la opinión pública impulsa el desarrollo normativo y jurisprudencial, incide en escenarios de toma de decisiones judiciales, promueve la visibilidad de la sociedad civil y también permite el acceso al conocimiento sobre las diversas tipologías de violencias y las formas o métodos que se pueden seguir para atentar contra la integridad del ser humano.

\section{METODOLOGÍA}

El desarrollo de esta investigación contempló la implementación de una metodología de investigación cualitativa comprendida desde propósitos de estudio descriptivos, que permitieron identificar impactos de la esfera mediática en los casos de agresiones con ácido a mujeres en España y Colombia.

De acuerdo con Yin (1994) el método de estudio de caso como método de investigación científica es apropiado en el abordaje de problemas sociales, teniendo en cuenta que permite examinar fenómenos contemporáneos en su entorno, el uso de múltiples fuentes de datos y el estudio de un caso único o múltiples casos.

Es en este sentido, la revisión normativa y documental realizada hizo posible la realización de uno de los principales objetivos de la investigación: la identificación y elección de dos casos paradigmáticos a partir de la recolección de información noticiosa relacionada con agresiones con agentes químicos -ácidos-, en medios de comunicación escrita de España y Colombia.

El período de tiempo comprendido para esta labor tomó como rango de estudio la búsqueda y documentación de los hallazgos entre los años 2010 y 2015. Razón que obedece, principalmente, al aumento de las cifras de las agresiones en Colombia y al surgimiento de las primeras iniciativas de orden legislativo en la materia, impulsadas por supuesto, por la agenda mediática característica en el tratamiento de esta problemática como un tema de interés público.

Así, la metodología de estudio de caso hizo posible identificar impactos de la esfera mediática en escenarios en los que el desarrollo del marco normativo y jurisprudencial de las agresiones con ácido, evidencian la interrelación de los elementos que integran la interpretación del contenido de la ley y la función social de las decisiones judiciales en la agenda pública. 
De esta forma, de acuerdo con Chetty (citado en Carazo 2006), el método de estudio de caso es una metodología que permite estudiar temas en los que las teorías existentes son insuficientes en el abordaje de fenómenos en los que se busca dar respuesta a cómo y por qué ocurren desde múltiples perspectivas. Es por lo que en el tema de estudio la opinión pública desempeña un papel de autoridad para la interpretación de la realidad, ha sido esta la que precisamente ha permitido desarrollos normativos importantes en la estructuración de tipos penales y decisiones judiciales en la materia.

\section{CASOS DE ESTUDIO}

Como se mencionó previamente, la elección metodológica tuvo como motivación principal la documentación de dos casos de mujeres sobrevivientes a agresiones con ácido en España y Colombia, en el período de tiempo comprendido entre el 2010 y 2015 .

El orden en el que se presentan, a continuación, expone parte de los hallazgos y de la documentación realizada a cada uno de ellos. Es importante señalar que el artículo presenta de manera más amplia la situación colombiana, pues es allí donde el impacto mediático en la agenda legislativa del país ha sido determinante en la tipificación de estos delitos.

\section{Caso español - María Ángeles Ruiz}

María Ángeles Ruiz es una mujer de nacionalidad española que contrajo matrimonio en el 2007 con Ozgur Dogan, hombre de nacionalidad turca, residente en España y quien planeó la agresión a María Ángeles al manifestar su intención de divorcio. Posteriormente, ante varios hechos que describe el caso, Ozgur Dogan contactó a un joven colombiano de nombre Bryan Stevan Cristancho, para que en un primer momento robara a María Ángeles mientras salía de su trabajo y para que, en un segundo momento, le lanzara ácido mientras ella caminaba por una de las calles de Madrid el 20 de junio de 2012. Como producto de esta última agresión, María Ángeles sufrió quemadura menor del 10\% de la superficie corporal localizada en la cara con una profundidad de tercer grado y una lesión corneal superficial en su ojo izquierdo.

Por estos hechos, Ozgur Dogan fue declarado autor de los delitos de coacciones graves, violencia física y psíquica habitual e inductor de los delitos de robo con violencia e intimidación y lesiones de gran deformidad con la agravante de alevosía, parentesco y disfraz. Por su parte, Bryam Stevan Cristancho, fue declarado autor de los delitos de robo con violencia e intimidación, malos tratos en el ámbito familiar y lesiones de gran deformidad, con la agravante de precio, alevosía y disfraz. Todos ellos contemplados en el Código Penal español. De esta forma, Ozgur Dogan y 
Bryan Stevan Cristancho son sentenciados a 18 y 13 años de prisión, respectivamente (Sentencia No. 252, 2014).

La documentación noticiosa de este caso permitió la recolección de noticias, entre las cuales se destacan las siguientes:

Tabla 1. Impacto del caso María Ángeles Ruiz en los medios de comunicación escrita de España

\begin{tabular}{|c|c|c|}
\hline Fecha & Titular de la noticia & Medio \\
\hline $2012 / 06 / 20$ & $\begin{array}{l}\text { Herida grave una mujer tras ser rociada por un hombre con áci- } \\
\text { do sulfúrico. }\end{array}$ & El País \\
\hline $2012 / 06 / 21$ & Marcada de por vida con ácido sulfúrico & La Razón \\
\hline $2012 / 06 / 22$ & $\begin{array}{l}\text { Cae el agresor que «roció» a María Ángeles. La Policía detiene } \\
\text { a un colombiano como autor del brutal ataque con ácido a la } \\
\text { joven }\end{array}$ & $A B C$ \\
\hline $2012 / 06 / 25$ & Caso de la mujer rociada con ácido: La decisión judicial. & La Razón \\
\hline 2014/03/14 & Piden 24 años por encargar que rociaran con ácido a su exmujer & $A B C$ \\
\hline 2014/03/21 & $\begin{array}{l}\text { "Ahora solo queda esperar que haya una sentencia justa". La } \\
\text { mujer atacada con ácido pide más medios contra la violencia } \\
\text { de género }\end{array}$ & El País \\
\hline 2014/04/10 & $\begin{array}{l}\text { Condenado a } 18 \text { años por encargar desfigurar a su mujer con } \\
\text { ácido }\end{array}$ & $A B C$ \\
\hline 2014/04/11 & 18 años de cárcel por encargar que echaran ácido a su esposa & El Pais \\
\hline $2014 / 04 / 27$ & La vida ya es bella para la chica del ácido & El Mundo \\
\hline 2015/02/03 & $\begin{array}{l}\text { El TS confirma la sentencia de } 18,6 \text { años de prisión para el } \\
\text { hombre que ordenó rociar con ácido a su mujer. }\end{array}$ & El Economista \\
\hline
\end{tabular}

Fuente: Elaboración propia.

Los criterios de selección de las noticias que integran el cuadro anterior obedecen principalmente al propósito de evidenciar la evolución del caso en la esfera mediática; evolución que será ampliada en la exposición de los resultados a través de la presentación de una línea de tiempo que identifica momentos clave en la agenda del caso.

\section{Caso colombiano - Natalia Ponce de León}

Natalia Ponce de León es una mujer colombiana que fue agredida con ácido el 27 de marzo de 2014 en la recepción del edificio donde se encontraba su vivienda en la ciudad de Bogotá. La tarde de aquel día, Natalia recibió una llamada donde anunciaron la presencia de su expareja, quien presuntamente la esperaba en la recepción. Natalia acude al llamado, pero en lugar de su expareja, se encontró con la presencia de Jonathan Vega, un conocido que, posteriormente sería identificado y judicializado, le roció ácido a través de una reja. 
Según el informe médico inicial, esta agresión le produjo a Natalia quemaduras en aproximadamente el 35\% del área de la superficie corporal total, comprometiendo su cara, labios, tórax, hombros, brazos, antebrazos, manos, dedos, muslos y ambas córneas (Soto, 2015).

Ante el alto impacto mediático del caso de la agresión de Natalia, se realizó un despliegue policial con el propósito de capturar al responsable. Jonathan Vega, el agresor de Natalia, era un hombre que, según varios testimonios, era un hombre que estaba obsesionado con ella desde varios años atrás. Por la agresión recibió luego de dos años una sentencia de 21 años de prisión.

La búsqueda de información noticiosa del caso Natalia Ponce arrojó un alto número de hallazgos, razón que orientó la documentación en la agresión, al seguimiento de la denuncia, la sentencia del agresor y el desarrollo legislativo que se adelantó desde el caso de Natalia orientado al aumento de penas y sanciones para los perpetradores de este tipo de delitos en Colombia.

A continuación, se relacionan algunos de los hallazgos encontrados que, como en el Caso María Ángeles, pretenden evidenciar la evolución del caso en la esfera mediática.

Tabla 2. Impacto del caso Natalia Ponce de León en los medios de comunicación escrita de Colombia

\begin{tabular}{|c|c|c|}
\hline Fecha & Titular de la noticia & Medio \\
\hline $2104 / 04 / 02$ & $\begin{array}{l}\text { Agresión con ácido a joven en Bogotá revive rechazo por vio- } \\
\text { lencia contra la mujer. }\end{array}$ & El País \\
\hline $2014 / 04 / 02$ & $\begin{array}{l}\text { El ácido en la cara y la política colombiana. } \\
\text { Recuperado de: FALTA LINK }\end{array}$ & Revista Semana \\
\hline 2014/04/06 & $\begin{array}{l}\text { Impunidad, la otra crueldad que sufren las víctimas del ácido. } \\
\text { Recuperado de: https:/goo.gl/m1gix2 }\end{array}$ & El Tiempo \\
\hline 2014/09/04 & $\begin{array}{l}\text { Ataques con ácido: ¿crimen de lesa humanidad? } \\
\text { Recuperado de: https://goo.gl/33A8w6 }\end{array}$ & Revista Semana \\
\hline 2015/04/11 & $\begin{array}{l}\text { El renacimiento de Natalia Ponce de León. } \\
\text { Recuperado de: https:/goo.gl/gxfnJB }\end{array}$ & El Tiempo \\
\hline $2015 / 11 / 25$ & $\begin{array}{l}\text { La ley por la que luchó Natalia Ponce de León. } \\
\text { Recuperado de: https://goo.gl/eCcrW5 }\end{array}$ & Revista Semana \\
\hline $2015 / 11 / 28$ & $\begin{array}{l}\text { 'La ley me da fuerzas para seguir luchando': Natalia Ponce de } \\
\text { León. Recuperado de: https://goo.gl/mhV2Ht }\end{array}$ & EL Tiempo \\
\hline 2015/12/02 & $\begin{array}{l}\text { Populismo e impunidad. } \\
\text { Recuperado de: https://goo.gl/zzLvMJ }\end{array}$ & El Espectador \\
\hline 2016/08/12 & $\begin{array}{l}\text { Caso Natalia Ponce: una tragedia que cambió las leyes. } \\
\text { Recuperado de: https://goo.gl/xos } 7 \mathrm{Xe}\end{array}$ & El Espectador \\
\hline $2016 / 12 / 14$ & $\begin{array}{l}\text { Jonathan Vega pagará } 21 \text { años de prisión por ataque a Natalia } \\
\text { Ponce. } \\
\text { Recuperado de: https://goo.gl/8cfuiN }\end{array}$ & El Tiempo \\
\hline
\end{tabular}

Fuente: Elaboración propia. 
Al igual que en el caso María Ángeles, la evolución de este en la esfera mediática será ampliada en la exposición de los resultados a través de la presentación de una línea de tiempo que identifica momentos clave en la agenda del caso.

\section{ANÁLISIS Y RESULTADOS}

El análisis de la información recolectada permitió plantear las siguientes líneas de tiempo y momentos en los cuales se desarrolló la agenda pública, legislativa y judicial de cada uno de los casos en la esfera mediática:

\section{Caso María Ángeles Ruiz}

La línea de tiempo planteada identificó cuatro momentos clave en los que la agenda pública se vio influenciada por la evolución del caso María Ángeles Ruiz en la esfera mediática española.

En un primer momento, para el 2012, los principales hallazgos hicieron referencia a la información noticiosa relacionada con la agresión y la detención de los perpetradores, uno de ellos como se expuso anteriormente, de nacionalidad colombiana.

Un segundo momento, se refiere a los hallazgos ausentes para el 2013; año determinante en el seguimiento a la recuperación de María Ángeles y al enjuiciamiento de los responsables.

El tercer momento, año 2014, es el que más hallazgos arrojó y corresponde a la información relacionada con la decisión judicial y a las reflexiones que algunos medios de comunicación hicieron a partir del caso de María Ángeles frente a la violencia de género en España y a columnas de opinión y crónicas.

El cuarto momento, refiere los únicos hallazgos encontrados para el 2015, correspondientes a la ratificación de la decisión judicial inicial por parte del Tribunal Supremo en lo Penal, frente a los recursos de casación interpuestos por los responsables. Vale la pena señalar, que a pesar de que esta decisión (Sentencia Desestimatoria No. 899), data de diciembre de 2014, solo tiene información noticiosa hasta febrero de 2015.

De manera complementaria, la línea de tiempo expuesta contempló, además de la evolución del caso en la esfera mediática, los principales medios de prensa escrita encargados del cubrimiento de la información noticiosa en cada uno de ellos. Se puede evidenciar que, en los años 2012 y 2014 la presencia de los principales medios de comunicación escrita en España estuvo presente en la documentación del caso; sin embargo, en los años 2013 y 2015 estuvieron totalmente ausentes.

\section{Caso Natalia Ponce de León}

La línea de tiempo planteada en la evolución del caso -Natalia Ponce de León-en la esfera mediática colombiana identificó tres momentos clave en la agenda pública. 
Un primer momento, corresponde al 2014, en el cual se identifican hallazgos que hacen referencia a la información noticiosa relacionada con la agresión, posterior identificación y detención del responsable de la agresión y adicionalmente, a partir de las supuestas motivaciones del agresor, los medios de comunicación producen información relacionada con su perfil psicológico. De forma complementaria, los hallazgos describen otros casos de agresiones con ácido cometidos en Colombia.

Un segundo momento, año 2015, refiere hallazgos sobre el proceso judicial adelantado contra Jonathan Vega y paralelamente se encuentran hallazgos sobre la recuperación de Natalia, seguimiento al trámite del Proyecto de Ley "Natalia Ponce de León".

El tercer momento, año 2016, presenta principalmente hallazgos sobre la sanción de la Ley 1773 de 2016, más conocida como "Ley Natalia Ponce de León", la cual hoy en día eleva las penas y sanciones a los agresores e incrementa el control ante la comercialización de sustancias químicas en Colombia. Adicionalmente, el año 2016 refleja un amplio cubrimiento a la Sentencia proferida contra el agresor de Natalia, Jonathan Vega.

Como se dijo en el caso María Ángeles, la línea de tiempo expuesta para el caso de Natalia también contempló la identificación los principales medios de prensa escrita encargados del cubrimiento de la información noticiosa en los momentos de la evolución de su caso en la agenda pública.

Figura 1. María Ángeles Ruiz

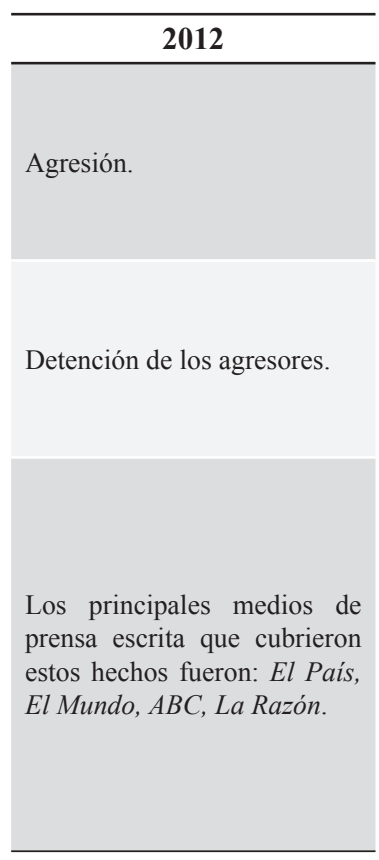

\begin{tabular}{|c|}
\hline 2014 \\
\hline $\begin{array}{l}\text { Decisión judicial sobre agre- } \\
\text { sores. }\end{array}$ \\
\hline $\begin{array}{l}\text { Crónicas sobre el caso de Ma- } \\
\text { ría Ángeles. }\end{array}$ \\
\hline $\begin{array}{l}\text { Columnas de opinión y re- } \\
\text { flexiones sobre violencia de } \\
\text { género. }\end{array}$ \\
\hline $\begin{array}{l}\text { Los principales medios de } \\
\text { prensa escrita que cubrieron } \\
\text { estos sucesos fueron: El País, } \\
\text { La Razón, } A B C \text {. }\end{array}$ \\
\hline
\end{tabular}

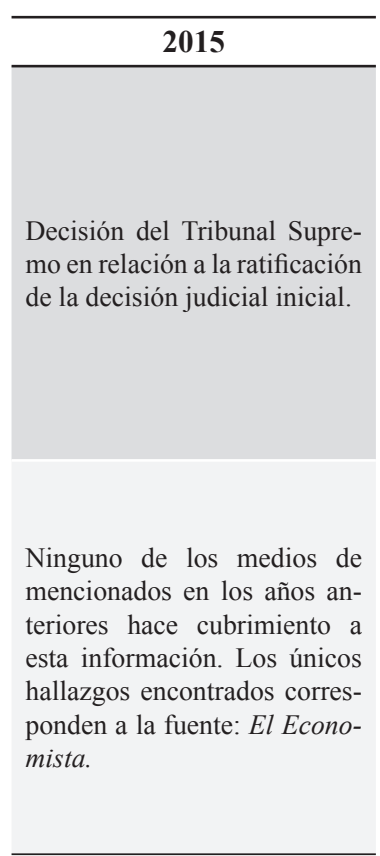


Figura 2. Natalia Ponce de León

\begin{tabular}{l}
\hline \multicolumn{1}{c}{$\mathbf{2 0 1 4}$} \\
\hline Agresión. \\
\hline $\begin{array}{l}\text { Identificación y detención del } \\
\text { agresor. }\end{array}$ \\
Perfil psicológico, motivacio- \\
nes y contexo del agresor \\
$\begin{array}{l}\text { Descripción de otros casos de } \\
\text { agresiones. }\end{array}$ \\
$\begin{array}{l}\text { Los principales medios de } \\
\text { prensa escrita usados en la } \\
\text { documentación de este suceso } \\
\text { fueron: El Tiempo, Revista Se- } \\
\text { mana, El Espectador, El País. }\end{array}$ \\
\hline
\end{tabular}

\begin{tabular}{l}
\hline \multicolumn{1}{c}{2015} \\
\hline $\begin{array}{l}\text { Proceso judicial contra Jo- } \\
\text { nathan Vega. }\end{array}$ \\
Recuperación de Natalia. \\
Creación de Fundación Nata- \\
lia Ponce de León. \\
Seguimiento al trámite del \\
Proyecto de Ley. \\
Los principales medios de \\
prensa escrita usados en la \\
documentación de este suceso \\
fueron: El Tiempo, Revista Se- \\
mana, El Espectador, El País. \\
\hline
\end{tabular}

\begin{tabular}{l}
\hline \multicolumn{1}{c}{2016} \\
\hline Sanción de la Ley 1773 de \\
2016 "Ley Natalia Ponce de \\
León". \\
Iniciativas de la Fundación \\
Sentencia Jonathan Vega \\
Los principales medios de \\
prensa escrita usados en la \\
documentación de este suceso \\
fueron: El Tiempo, Revista Se- \\
mana, El Espectador, El Pais. \\
\hline
\end{tabular}

\section{DISCUSIÓN Y CONCLUSIONES}

\section{Contexto internacional normativo de las agresiones con ácido}

Países como Bangladesh, Camboya e India han adoptado diversas normativas internas con el propósito de castigar los ataques con agentes químicos que desde los años 90 vienen incrementando sus estadísticas. Bangladesh en el 2002 elevó los castigos por estas agresiones a pena de muerte, a través de la Ley Acid Crime Control Act (ACCA) en los casos en los que se genera daños en rostro, pecho, órganos sexuales, pérdida de la visión o audición. En este país la mayoría de los casos corresponden a violencia de género perpetrada a niñas y mujeres y a disputas por el control de tierras (Soto, 2015).

En Pakistán, la Criminal Law Amendement Act, aprobada en el 2011, contempla la cadena perpetua para este tipo de agresiones y el endurecimiento de penas ante los hechos de discriminación y prácticas misóginas. Camboya, por su parte, promulgó en el 2012 una ley que también aumenta las penas para los agresores y que a la vez contempla la obligación y responsabilidad del Estado en la prestación de servicios médicos y jurídicos y en la regulación del comercio de estas sustancias. Sin embargo, en países como Irán además de contemplarse la pena de muerte, se exige que en la 
mayoría de los casos al agresor se le apliquen gotas de ácido directamente sobre los ojos, exceptuando los casos en los que por solicitud de la víctima se exija el pago de una multa que sea destinada a su rehabilitación; o en el caso de India, en el que la víctima pide que el agresor reciba un daño físico equivalente, además de la aplicación de una pena no inferior a diez años y que según los daños causados puede llegar a cadena perpetua (Soto, 2015).

En España, a pesar de que esta situación no es tan frecuente, se cuenta con un marco normativo que atiende a las medidas de protección integral contra la violencia de género a través de leyes como la Ley Orgánica 1 del 28 de diciembre de 2004, la Ley Orgánica del Poder Judicial y la Ley de Enjuiciamiento Criminal, por medio de las cuales, como en el caso de María Ángeles, se sancionan penalmente las agresiones con ácido como delitos de gran deformidad.

En Colombia, ante el evidente aumento de casos, el 2 de julio de 2013 se promulgó la Ley 1639 como la primera iniciativa para castigar este tipo de delitos. Sin embargo, ante el alto impacto mediático del caso de agresión ocurrida a Natalia Ponce de León, el 27 de marzo de 2014, una nueva iniciativa tuvo eco en la legislación colombiana. Así, en enero de 2016 se promulga la Ley "Natalia Ponce" - Ley 1773 de 2016, que eleva las penas a los agresores, condiciona sus beneficios e incrementa el control y las sanciones ante la comercialización y distribución de sustancias químicas en el país.

\section{Reflexiones sobre la situación en España y Europa}

Como se mencionó previamente, en España las agresiones con ácido no son una situación constante. Sin embargo, resulta importante señalar que información noticiosa reciente evidencia la existencia de nuevos casos y el aumento significativo de las cifras en otros países del continente europeo.

Información de diarios como El Mundo, El Colombiano y La Capital, evidencian el aumento de estas agresiones en países como Inglaterra que, con una población aproximada a los 65 millones de habitantes, registró, en el 2016, 720 casos de agresiones (394 de ellas solo en Londres); es decir, un estimado de dos casos por día (Rodríguez, 2017).

En España, al caso de María Ángeles se suman dos casos ocurridos en 2014 y 2017; el primero de ellos un gallego doctor en física, rociado por error en Valencia, quien sufrió pérdida total del ojo izquierdo, pérdida de visión parcial del ojo derecho y transformación persistente de la personalidad. El segundo, corresponde al de una mujer que lanzó ácido a la cara de una vecina en Logroño. La mujer agredida padeció quemaduras de primer y segundo grado en el cuello, rostro, lengua, brazos y pecho (Mucha, 2017).

Finalmente, en el contexto de la situación en España y en el continente europeo, es importante resaltar esfuerzos e iniciativas que, desde organizaciones civiles, como Sigo Adelante y Acid Survivors Trust International - ASTI, se adelantan por 
contribuir al restablecimiento de los derechos de sobrevivientes a estas agresiones, especialmente mujeres, de su nacionalidad y de otros países.

\section{Reflexiones sobre la situación en Colombia}

El primer caso de agresión con agente químico reportado en Colombia data en el año 1996. Ginna Potes, sobreviviente a esta agresión, es actualmente una de las principales activistas que, desde "Reconstruyendo Rostros", la organización civil que lidera, acompaña los procesos de reparación y acceso a la justicia de diferentes sobrevivientes y sus familias. El aumento de casos desde esta fecha y el impacto mediático de otros como el de Natalia Ponce de León, ocurrido en el 2014, promovieron el haber establecido medidas políticas y jurídicas encaminadas a erradicar los ataques con agentes químicos, especialmente hacia las mujeres.

Estadísticas de estas agresiones arrojaron para el 2014 una cifra total de 982 casos, 565 de ellos ocurridos a mujeres. Para mediados del 2013 el Instituto Nacional de Salud informó que solamente entre julio de 2012 y el primer trimestre de 2013, se registraron 93 ataques con sustancias químicas en el país, resaltando que de estas cifras el $83.3 \%$ de las víctimas eran mujeres, al tiempo que el $79.5 \%$ de los agresores eran hombres (Secretaría Distrital de la Mujer, 2012).

Según las cifras del Instituto Nacional de Medicina Legal y Ciencias Forenses, los números de la siguiente gráfica corresponden a los casos atendidos por el Instituto, cifras que, al no contemplar los casos o ataques no reportados, no permiten tener estadísticas claras y homogéneas sobre el número real o aproximado total de casos de agresiones con agentes químicos en Colombia.

Figura 3. Agresiones con ácido a mujeres atendidas por el Instituto Nacional de Medicina Legal

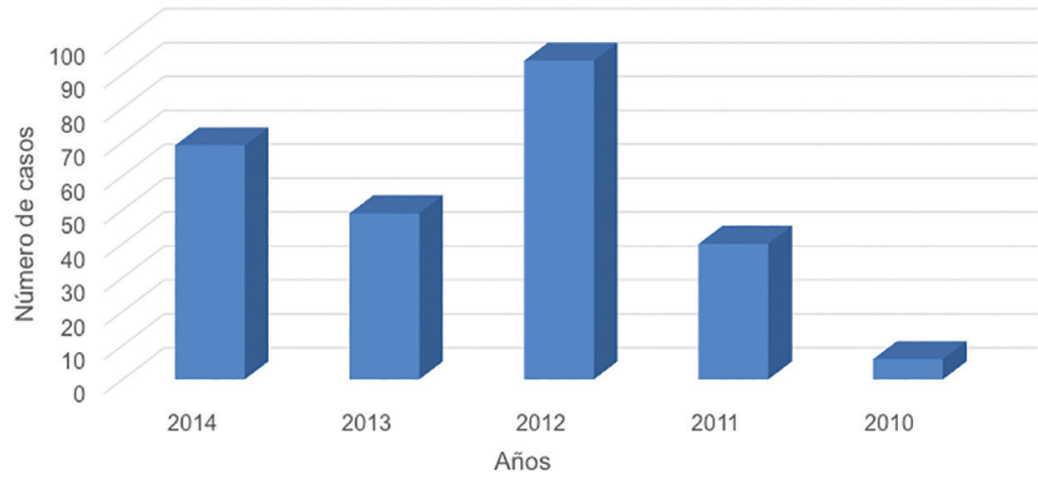

Fuente: Medina y Acosta, 2014, pp. 61- 87. 
Como se ha manifestado previamente, ante esta problemática, el Estado colombiano ha realizado ajustes en su legislación adoptando medidas penales y de control, a partir de la expedición de normas referentes a la prevención, protección especial, atención integral para las víctimas e incorporación de sanciones para quienes incurran en estos delitos. Medidas que contribuyen, además, a un efectivo control y registro de la información estadística, identificado como uno de los principales problemas en la documentación de la temática.

Versiones no positivistas de autores, como Mauricio García Villegas, acercan a la comprensión de diversas problemáticas sociales en las cuales el Estado y la sociedad son los principales protagonistas en el incumplimiento de las reglas. Sus reflexiones acerca de este comportamiento llevan a identificar aciertos, al considerar el desacato de las normas como causa de la violencia y su relación con el desarrollo económico de las naciones, si bien, como se mencionó inicialmente, las agresiones con ácido se presentan principalmente en países con culturas y costumbres particulares, bien, podría afirmarse que la mayoría de ellos pueden ser considerados países en vías de desarrollo.

De acuerdo con los postulados de García (2009, p. 21), la ausencia de instituciones estatales y, en particular, la falta de una justicia penal eficiente y capaz de disuadir a los delincuentes, es la causa de la extraordinaria reproducción del crimen que tuvo lugar en Colombia a finales del siglo XX, época que corresponde con el auge del narcotráfico y de delitos como las agresiones con ácido.

Podría pensarse que tal vez estos últimos no tienen una relación común, sin embargo, fue precisamente el narcotráfico uno de los principales escenarios para llevar a cabo estas prácticas, ya sea como insumo en la producción de sustancias estupefacientes, modo de tortura, o como mecanismo de evasión de la justicia al ser usado para borrar las huellas dactilares que permitían la identificación de los delincuentes.

Es relevante reconocer que diferentes prácticas mediáticas que han caracterizado el tratamiento noticioso de estas agresiones, resultan en la difusión de la información sobre el acceso y comercialización de estas sustancias; las cuales, desde una esfera pública mediatizada, han posicionado en las agendas públicas actividades delictivas como el narcotráfico, el cual ha marcado en Colombia el inicio de una práctica que en los últimos años se ha convertido en una de las formas más crueles y violentas de agresión contra la integridad del ser humano.

Autores sobre los que García (2009, p. 22) llama particularmente la atención, como Francisco Thoumi, quien atribuye la falta de sanciones como la causa principal del florecimiento de la ilegalidad en Colombia, refleja no solo la debilidad del Estado sino la de la sociedad al tolerar la ilegalidad. El incumplimiento de las reglas en la compra, venta, distribución y comercialización de las sustancias químicas es un claro ejemplo de la debilidad de la justicia y la incapacidad estatal para erradicar este tipo de actividades criminales. 
Esta debilidad se hace evidente en casos particulares en los que se genera movilización social frente a violaciones, asesinatos y casos de violencia extrema. Casos como los de Rosa Elvira Cely, víctima de feminicidio, y Natalia Ponce de León, son claros ejemplos de la influencia de los medios de comunicación en el tratamiento social, jurídico y judicial de estas situaciones. Son casos, en los que el alcance mediático es determinante en los fallos judiciales y en las decisiones legislativas en la materia.

Si bien, estos casos no son los únicos reportados en las cifras de las estadísticas de este tipo, sí han sido determinantes en la reglamentación de tipos penales ante estas agresiones. Leyes conocidas comúnmente como "Ley Rosa Elvira Cely" y "Ley Natalia Ponce de León", en su orden: Ley 1761 del 6 de julio de 2015, por la cual se crea el tipo penal de feminicidio como delito autónomo y Ley 1773 del 6 de enero de 2016, por la cual se aumentan las penas y las sanciones frente a las agresiones con ácido a mujeres en Colombia, son clara evidencia de los impactos de la esfera mediática en los asuntos públicos.

Puede pensarse, tal vez, que estas iniciativas surgen ante los ideales del garantismo penal al que hace referencia Ferrajoli (citado en Moreno, 2007) o al fanatismo del legalismo que menciona García (2009). Sin embargo, lo que es evidente reconocer es la influencia de los medios de comunicación en la movilización social, en la tipificación de estos delitos y en el empoderamiento de poblaciones de víctimas y sobrevivientes a estas agresiones.

Ricardo Medina, penalista y académico colombiano, señala que el dinamismo de la sociedad, caracterizado por la influencia mediática, ha llevado a que se permee una dogmática jurídica estable, adaptada a diversos intereses políticos, sociales o personales. El autor manifestó, antes de la tipificación del delito, que se debían abordar penalmente las agresiones con ácido, luego de contemplar las diferentes figuras jurídicas bajo las cuales se ha caracterizado esta situación (2014).

\section{CONSIDERACIONES FINALES}

El abordaje de los casos de estudio elegidos en la investigación permite identificar impactos de la esfera mediática en las agresiones con ácido a mujeres en España y Colombia en varios frentes:

\section{Impactos legislativos y judiciales}

Como se quiso hacer evidente en el documento, uno de los principales impactos del tratamiento noticioso de las agresiones con ácido resulta en la tipificación de estos delitos y en la estructuración de normas que regulan la venta y comercialización de sustancias químicas.

Sistemas jurídicos como el colombiano, se han visto desarrollados a través de la implementación de medidas tendientes a la sanción de los responsables de 
estos delitos; sin embargo, a pesar de estas iniciativas los datos y la información noticiosa reportada evidencia que no han sido vías definitivas en la erradicación de estas prácticas, en su lugar, los casos siguen apareciendo, y tanto víctimas como sobrevivientes sufren vulneraciones en sus derechos, pierden garantías en el acceso a la justicia y reciben una precaria atención en materia de salud.

El desarrollo de herramientas propias del litigio estratégico y la defensa del interés público, son determinantes en la consolidación de políticas de defensa y promoción de los derechos humanos. Según lo expuesto por Londoño Toro et al, en su libro Educación Legal Clínica y defensa de los Derechos Humanos (2009), la elección de casos de alto impacto, permite materializar el seguimiento a las decisiones judiciales desde la interdisciplinariedad a través de la conjugación de herramientas jurídicas, políticas y sociales que, evidenciadas en la consolidación de alianzas estratégicas, contemplan actores y recursos determinantes en la defensa de los derechos humanos, como los medios de comunicación.

\section{Fortalecimiento de organizaciones civiles}

Otro de los impactos de la esfera mediática en las agresiones con ácido, resulta en la estructuración y fortalecimiento de organizaciones civiles que, lideradas en su mayoría por sobrevivientes a estas agresiones, adelantan acciones encaminadas a la garantía y reivindicación de sus derechos. El desarrollo de iniciativas de investigaciones conjuntas y trabajos de extensión de la mano de la academia ha permitido la presentación de productos importantes que abordan temáticas como los derechos de las sobrevivientes, atención y primeros auxilios en salud y protocolos para la investigación y sanción de estas agresiones.

Ejemplo de esto son los resultados de proyectos como "Propuesta pedagógica: Reparación integral y justicia para las víctimas de ataques con agentes químicos", que con el liderazgo de la academia han hecho posible la existencia de convenios interinstitucionales y el trabajo conjunto con fundaciones como "Reconstruyendo rostros" y "Natalia Ponce de León en Colombia", y "Sigo adelante", en España; los cuales han permitido empoderar a la población y propiciar espacios de diálogo e interacción entre sobrevivientes, operadores judiciales y personal de salud, para establecer canales de apoyo efectivos en el restablecimiento de los derechos de esta població

\section{Importancia de protocolos en medios de comunicación y su valor como actor de educación informal}

Como ha sido posible identificar, el abordaje de las agresiones con ácido en la esfera mediática y en la agenda pública centra su atención en el desarrollo de los procesos legislativos, judiciales y en el restablecimiento y garantía de los derechos de víctimas y sobrevivientes, como el acceso a la salud y el acceso a la justicia. 
Sin embargo, este escenario también ha permitido que se omita la importancia de un uso adecuado del lenguaje y de la imagen, el cual, ante inmediatez que caracteriza el ejercicio periodístico, en la mayoría de los casos resulta en la revictimización de esta población.

Los medios de comunicación deben contemplarse como un instrumento de educación informal que permita el acceso a la información y que haga posible la participación y el acceso a la justicia. La legitimación de prácticas y la repetición de acciones particulares, que una vez aceptadas por parte de la sociedad justifican el incumplimiento de las normas y son causales, no solo de costumbres sino de delitos, condicionan además de la conducta de la sociedad, la prevalencia del individualismo, el cumplimiento de las normas desde su inobservancia y la legitimidad de la autoridad encargada de la garantía de la convivencia, el desarrollo, la justicia y la democracia.

Reconocer los impactos de la esfera mediática en el tratamiento de asuntos públicos y problemáticas como las agresiones con ácido, el reconocimiento de su valor y uso pedagógico, fortalecen espacios que contribuyen eficazmente no solo en la expedición de normativas, sino a la disminución y erradicación de nuevos casos de violencia.

Por estas razones, este documento llama la atención sobre la importancia de la reflexión académica y social de esta problemática, reconociendo su incidencia nacional, internacional y las implicaciones mediáticas de estas agresiones en la esfera pública, en el desarrollo normativo y jurisprudencial de los Estados y en la construcción de una cultura de derechos, a partir de su abordaje como problema de interés público.

\section{APOYOS}

Este documento presenta resultados de investigación conseguidos en el desarrollo del proyecto "Estudio comparado de los impactos normativos y mediáticos de las agresiones con ácidos a mujeres sobrevivientes en España y Colombia (2010-2015)", financiado por el Programa de Becas del Ministerio de Asuntos Exteriores y de Cooperación de España y la Agencia Española de Cooperación Internacional para el Desarrollo - MAEC/AECID (2015-2016). De forma complementaria, este trabajo corresponde con el proceso de formación adelantado en el Programa de Maestría en Derecho con énfasis en Derechos Humanos de la Universidad del Rosario, Colombia, acompañado de manera permanente por docentes e investigadoras de la Universidad de Málaga en España y la Universidad del Rosario en Colombia.

\section{REFERENCIAS}

ABC. (2012, julio 19). Cae el agresor que «roció» a María Ángeles. $A B C$, p. 49.

ABC. (2014, marzo 14). Piden 24 años por encargar que rociaran con ácido a su exmujer. $A B C$, p. 95. 
Barroso, J. (2012, junio 20). Herida grave una mujer tras ser rociada por un hombre con ácido sulfúrico. Recuperado de https://goo.gl/m26zeB

Barroso, J. (2014, marzo 21). "Ahora solo queda esperar que haya una sentencia justa". La mujer atacada con ácido pide más medios contra la violencia de género. El Pais, p. 33.

Carazo, P. M. (2006). El Método de estudio de caso. Estrategia metodológica de la investigación científica. Pensamiento y Gestión, 165-193.

Colprensa y Redacción de El País Bogotá y Cali. (2014, abril 2). Agresión con ácido a joven en Bogotá revive rechazo por violencia contra la mujer. Recuperado de https://goo.gl/N72TNJ

Congreso de la República. (2015). Ley 1761 del 6 de julio de 2015. Colombia.

Congreso de la República. (2016). Ley 1773 del 6 de enero de 2016. Colombia.

Declaración sobre la eliminación de la violencia contra la mujer. (20 de diciembre de 1993). Recuperado de http://www.ohchr.org/SP/ProfessionalInterest/Pages/ ViolenceAgainstWomen.aspx

El Economista. (2013, febrero 3). El TS confirma la sentencia de 18,6 años de prisión para el hombre que ordenó rociar con ácido a su mujer. Recuperado de https:// goo.gl/AUWv55

El Espectador. (2015, diciembre 2). Populismo e impunidad. Recuperado de https:// goo.gl/zzLvMJ

El Mundo. (2014, abril 27). La vida ya es bella para la chica del ácido. El Mundo, pp. $10-12$.

El País. (2014, abril 11). 18 años de cárcel por encargar que echaran ácido a su esposa. El País, p. 35.

El Tiempo. (2014, abril 6). Impunidad, la otra crueldad que sufren las víctimas del ácido. Recuperado de https://goo.gl/m1gix2

El Tiempo. (2015, mayo 12). El renacimiento de Natalia Ponce de León. Recuperado de https://goo.gl/gxfnJB

El Tiempo. (2015, noviembre 28). 'La ley me da fuerzas para seguir luchando': Natalia Ponce de León. Recuperado de https://goo.gl/mhV2Ht

El Tiempo. (2016, diciembre 14). Jonathan Vega pagará 21 años de prisión por ataque a Natalia Ponce. Recuperado de https://goo.gl/8cfuiN

García, M. (2009). Introducción: La cultura del incumplimento de reglas. En M. García. Normas de papel. La cultura del incumplimiento de reglas (pp. 237 307). Bogotá: Siglo del Hombre Editores.

Instituto Nacional de Estadísitica. (2017, mayo 31). Estadística de violencia doméstica $y$ violencia de género. Recuperado de http://www.ine.es/dyngs/INEbase/es/ operacion.htm?c=Estadistica_C\&cid=1254736176866\&menu=ultiDatos\&i $\mathrm{dp}=1254735573206$ 
La Razón. (2012, junio 25). Caso de la mujer rociada con ácido: La decisión judicial. La Razón, p. 46.

La Razón. (2012, junio 21). Marcada de por vida con ácido sulfúrico. Recuperado de https://goo.gl/rxy9ak

Londoño, B. (2009). Educación legal clínica y defensa de los derechos humanos. Bogotá: Editorial Universidad del Rosario.

Londoño, B., Rubio, L., \& Castañeda, J. F. (2017). La violencia de género no tiene fronteras. Estudio comparativo de las normativas colombiana y española en materia de violencia de género (2004-2014). Revista Derecho del Estado, 127154.

Moreno, R. (2007). El modelo garantista de Luigi Ferrajoli. Lineamientos Generales. Boletin Mexicano de Derecho Comparado, nueva serie, año XL, núm.120, septiembre - diciembre de 2007.

Mucha, M. (2017, julio 9). Europa bajo ataque de ácido. El Mundo.

Revista Semana. (2014, septiembre 4). Ataques con ácido: ¿crimen de lesa humanidad? Recuperado de https://goo.gl/33A8w6

Revista Semana. (2014, abril 2). El ácido en la cara y la política colombiana. Recuperado de https://goo.gl/mQ7PAC

Revista Semana. (2015, noviembre 25). La ley por la que luchó Natalia Ponce de León. Recuperado de https://goo.gl/eCcrW5

Rodolfo, C. M. (2007, diciembre). El modelo garantista de Luigi Ferrajoli. Lineamientos generales. Boletín Mexicano de Derecho Comparado.

Rodríguez, C. (2017, julio 9). La ciudad del Támesis, capital mundial del ácido. El Mundo.

Rubiano, M. P. (2016, agosto 12). Caso Natalia Ponce: una tragedia que cambió las leyes. Recuperado de https://goo.gl/xos7Xe

Secretaría Distrital de la Mujer. (2012). Boletín Informativo.

Sentencia Desestimatoria No. 899, Recurso de casación (P) No. 10486/2014 P (Tribunal Supremo. Sala de lo Penal 26 de 12 de 2014).

Sentencia No. 252, PO 14/2013 (Juzgado de Violencia sobre la Mujer No. 3 de Madrid 04 de 04 de 2014).

Soto, M. (2015). El renacimiento de Natalia Ponce de León. Itinerario de una vida que venció a la barbarie. Bogotá: Intermedio Editores.

Villalobos, C. A. (2017). Los ataques con agentes químicos como una forma de violencia extrema contra las mujeres en Colombia. Revista Temas Socio Jurídicos, 13-33.

Yin, R. (1994). Case Study Research. Design and Methods. Applied Social Research Methods (vol. 5). Londres: Sage Publications. 